Review Article

\title{
Association of TLR-2 Gene Polymorphisms with the Risk of Periodontitis: A Meta-Analysis
}

\author{
Chao Shan $\left(\mathbb{D}\right.$, Abasijiang Aisaiti $\left(\mathbb{D}\right.$, Zhong Peng Wu $\left(\mathbb{D}\right.$, Ting Ting Wang $\mathbb{D}^{\circ}$, and Jin Zhao \\ Department of Endodontics, First Affiliated Hospital of Xinjiang Medical University (Affiliated Stomatological Hospital), \\ Urumqi 830054, China \\ Correspondence should be addressed to Jin Zhao; merryljin@sina.com
}

Received 16 January 2020; Revised 26 May 2020; Accepted 22 July 2020; Published 8 August 2020

Academic Editor: Roberta Rizzo Copyright ( $) 2020$ Chao Shan et al. This is an open access article distributed under the Creative Commons Attribution License,
which permits unrestricted use, distribution, and reproduction in any medium, provided the original work is properly cited.

\begin{abstract}
Background. Periodontitis is a kind of chronic infectious disease, affecting the health of billions of people. In recent years, a number of studies have shown that multiple immune gene polymorphisms are associated with the susceptibility to periodontitis, among which TLR-2 plays a critical role in periodontitis. But most of the studies reported TLR-2 gene polymorphism and susceptibility to periodontitis are not consistent. Therefore, we included all eligible studies in our study for further meta-analysis. Methods. We used electronic databases, including CNKI, PubMed, EMBASE, and Web of Science databases, and relevant research published through June, 2020. Selecting studies involved case-control trials. For all eligibility studies, odds ratios (ORs) and 95\% confidence intervals (95\% CI) are provided or can be calculated from the study data. The size of the combined effect was calculated using STATA 15.0. Results. Our meta-analysis included 14 articles representing 18 case-control studies with a total of 3873 cases and 3438 control subjects. Significant association was found between periodontitis and TLR-2 rs1898830 polymorphism under the allelic model (A allele vs. $\mathrm{G}$ allele: $p=0.014, \mathrm{OR}=1.208,95 \% \mathrm{CI}: 1.039-1.406$ ), recessive model (GG vs. GA+AA: $p=0.028, \mathrm{OR}=0.755$, 95\% CI: $0.588-0.970$ ), and codominant model (GG VS. AA: $p=0.014$, OR $=0.681,95 \%$ CI: 0.501-0.925). In subgroup analysis, TLR-2 rs5743708 polymorphism was associated with periodontitis risk in Asians under an allelic model (G allele vs. A allele: $p=0.017, \mathrm{OR}=12.064,95 \% \mathrm{CI}: 1.570-92.688$ ), dominant model (GA+AA vs.GG: $p=0.016, \mathrm{OR}=0.08,95 \% \mathrm{CI}$ : 0.010-0.620), and codominant model (GA VS. GG: $p=0.016, \mathrm{OR}=1.026,95 \%$ CI: 0.821-1.282). Conclusion. The TLR-2 rs1898830, rs5743708 polymorphism may be associated with susceptibility to periodontitis. In the future, genome-wide approaches and large-scale, multiethnic case-control trials are still needed.
\end{abstract}

\section{Introduction}

Periodontitis is a chronic infectious disease caused by the accumulation of bacteria such as P.g (Porphyromonas gingivalis) and A.a (Aggregatibacter actinomycetemcomitans), which leads to the destruction of the surrounding periodontal tissue, including deep pockets, loss of attachment, and loss of the alveolar bone $[1,2]$. Moreover, it is the main cause of adult tooth loss, which requires huge expenditure for the prevention and treatment of periodontal disease every year $[3,4]$. Currently, the idea that plaques and microorganism's well-characterized shift-the change from the gram-positive to the gram-negative was in the process of the change from periodontal health to periodontal disease-are the initial factors of periodontitis. P.g, Tannerella forsythia, and Treponema denticola are designated the "red-complex" periopathogens and have been studied extensively [5]. A large number of previous studies have confirmed that both periodontitis are caused by a complex combination of bacterial infection and genetic factors, with heritability estimates as high as $50 \%$ [6]. Unlike some monogenic diseases usually caused by a single gene, genetic susceptibility to periodontitis can be determined by hundreds of genes, while clinical phenotypes are determined by the interaction of environmental, genetic, and epigenetic factors [7]. However, the most known or assumed genetic risk loci for chronic periodontitis (CP) are based on candidate gene studies; these small effect genes have small to moderate effects and explain only a small fraction of disease heritability for periodontitis. Recently, some studies have 
focused on genome-wide approaches rather than candidate gene approaches, thus considering the possibility that multiple genes may be causal factors for periodontitis [8-10].

Toll-like receptors (TLRs) are kinds of pattern recognition receptors (PRRs), which can be specific to identify pathogenic microorganisms that are conservative antigen molecules in evolution, such as lipopolysaccharide (LPS), which are the main components of the outside membrane of gram-negative bacteria, lipoproteins, or other parts of bacterial or fungal cell wall components, causing the host production of inflammatory cytokines and chemokines such as IL-18 and IL-1 $\beta$ [11]. Thus, PRRs monitoring the invasion of pathogenic microorganisms effectively induce the immune response and regulate innate inflammation [12]. The role of TLR-2 in the pathophysiology of periodontitis has been widely discussed; elevated levels of TLR-2 were found in gingival inflammatory tissue [13]. In addition, knockout experiments in mice suggested that TLR2 identifies the key periodontal bacteria like P.g and A.a in periodontal infection [14]. In Scheres et al.'s study, P.g's LPS was used to stimulate human gingival fibroblasts in vitro, causing higher expression levels of TLR1, TLR2, and TLR7 [15].

Interestingly, although numerous studies have demonstrated a link between TLR-2 and periodontitis susceptibility, focusing on certain SNPs like rs5743708 and rs1898830, they showed no positive conclusion from their findings. This could be due to a small sample size and incomplete capture of genetic variation, or previous studies focused only one or several SNPs. Genetic association studies designed to examine the relationship between genetic variation and complex results must be treated with caution, even if the results are negative, as many factors may affect the results [16]. The meta-analysis is aimed at (1) overcoming the shortage of small sample research, (2) conducting a comprehensive analysis, (3) objectively evaluating statistical methods, and (4) comprehensively applying various effects to achieve the same goal. This approach leads to more reliable conclusions than a single study. In recent years, meta-analysis has been widely used in gene polymorphism research. Therefore, the meta-analysis and subgroup analysis further clarify the relationship between TLR2 polymorphism and periodontitis susceptibility in order to reconcile inconsistencies across individual studies.

\section{Materials and Methods}

2.1. Protocols and Eligibility Criteria. The meta-analysis and systematic review reported here are in accordance with the Preferred Reporting Items for the Systematic Review and MetaAnalyses (PRISMA) statement [17]. The literature search was limited to original studies performed in humans on the association of Toll-like receptor 2 SNPs with periodontitis risk.

2.2. Search Strategy. Data were collected from the CNKI (China National Knowledge Infrastructure), PubMed (PubMed is an interface for the Medline database), Embase, and Web of Science databases up to June, 2020 (last access on June, 2020). The search terms comprised the following 3 items and were combined using the Boolean operator AND: gene name (including "TLR-2", “Toll-Like Receptor 2" or “TLR 2" or "tlr-2" or "tlr-2"). genetic polymorphisms (including "genetic variant", "genetic "variation", "polymorphism", "mutation" or "mutan"), and disease (including "periodontal disease", "periodontitis", "chronic periodontitis", "CP" or "PD") to identify studies describing associations of genetic polymorphisms with periodontitis susceptibility. Relevant publications were also identified via searches supplemented by a literature review. Published articles in Chinese and English were retrieved. Moreover, hand searches for references cited in the published original and review articles were also performed.

2.3. Selection Criteria. Two authors identified studies eligible for further review by performing an initial screen of identified titles or abstracts. Articles considered for inclusion in the meta-analysis had to meet the following inclusion criteria: (a) studies used validated genotyping methods (such as PCR-RFLP or TaqMan) to measure the association of SNPs in TLR-2 genes with periodontitis risk; (b) a case-control cohort or nested case-control design; (c) the full text of the studies was available, and the data of the studies were not duplicated in another manuscript; (d) the case group of patients had a clinical diagnosis of periodontitis, including $\mathrm{CP}$ patients and aggressive periodontitis (AgP) patients, whereas the control group was the periodontal healthy population; and (e) when multiple document data were identical or overlapping, the most recently published paper was selected. Reviews, editorials, and case reports were excluded. Both reviewers fully agreed on the eligibility of the included articles in this first screening. Then, we performed a second screening based on full-text review. Studies such as cohort studies or nested case-control design analyses are included. According to the objective of this analysis, studies were excluded if they did not provide enough information on genotype frequency or did not report sufficient genotype distribution for the calculation of odds ratios (ORs) and its variance. Besides, studies were also excluded if the genotype distributions of control subjects were varied from Hardy-Weinberg equilibrium (HWE). Moreover, the case groups which suffered not only from periodontitis but also from other diseases were also excluded.

2.4. Data Extraction and Quality Assessment. We extracted detailed data on the first author's name, year of publication, region of the study, disease type, genotype, and smoking status. Furthermore, the evidence of HWE in controls was verified through the application of an online software (http://www.oege.org/software/hwe-mr-calc.shtml). $p$ value less than 0.05 of HWE was considered to be significant. Data extracted was performed independently by two investigators, and discrepancies were reviewed by a third reviewer. The quality of the observational studies was assessed using the Newcastle-Ottawa scale, and they were examined independently by two researchers (http://wwwohrica/programs/ clinical_epidemiology/oxfordasp). Briefly, a quality score (0-9) was generated according to a maximum of 1 star for each item on selection. And the quality of each study was assessed by using the following methodological components: (1) subject selection and (2) comparability of subject.

2.5. Heterogeneity. Between-study and between-subgroup heterogeneities were evaluated by calculating the $I^{2}$ statistic 
and the Cochrane $\mathrm{Q}\left(\chi^{2}\right)$ statistic, with a $p$ value of 0.10 set for significance of the test of heterogeneity. $I^{2}$, directly calculated from the $Q$ statistic, indicates the percentage of variability in effect estimates because of true heterogeneity, rather than sampling error. $I^{2}$ ranges from $0 \%$ to $100 \%$, with $0 \%$ indicating the absence of any heterogeneity. Although absolute numbers for $I^{2}$ are not available, values $<50 \%$ are considered to have low heterogeneity, and the effect is thought to be fixed. Conversely, when $I^{2}$ exceeds $50 \%$, then heterogeneity is thought to exist and the effect is random.

2.6. Statistical Analysis. We performed a detailed metaanalysis to evaluate the association between TLR-2 SNPs and periodontitis susceptibility. We used five models, including the allele model, codominant models, dominant model, and recessive model, and subgroup analyses were performed based on the case type and racial descent. All data were analyzed using Stata version 15.0. Odds ratios (OR) and 95\% CIs were calculated as the parameter of efficacy. $I^{2}>50 \%$ could suggest heterogeneity and suggest the random-effect model (DerSimonian-Laird) for the secondary endpoint. Otherwise, the fixed-effect model (Mantel-Haenszel) for the primary was used to calculate pooled ORs. Two-sided probability values of $<0.05$ were considered statistically significant. Potential publication bias of studies with different sample sizes was examined by visual inspection of funnel plots. This study is registered with PROSPERO, number CRD42020154093.

\section{Results}

3.1. Study Selection and Characteristics. Our search identified a total of 381 potentially relevant studies (Figure 1). No previous meta-analysis on this issue was identified. After removal of duplicate publications, 293 articles remained. By reviewing the title and abstract, 233 articles were excluded. The full text of the remaining 60 articles was reviewed in detail, and 46 of these were subsequently excluded. Therefore, fourteen articles representing eighteen case-control studies involving 3873 patients and 3438 control subjects were included in our meta-analysis [18-31]. Comprising $\mathrm{CP}$ and/or AgP were comprehensively assessed against the inclusion criteria. Two of the eligible articles that were written in Chinese other than English were retrieved and translated in order to avoid publication bias. We described the main features of the eligible papers. A total of 6 gene polymorphism studies were included in this meta-analysis (rs1898830, rs5743704, rs5743708, rs3804100, rs13150331, and rs12191786), and other SNPs of TLR-2 such as rs7696323 and rs5743709 were reported only in one study. About the ethnic issue, there were ten studies taking the Caucasian as the research object, while eight studies were contraposing the Asian population. Five studies were excluded because they were not in accordance with HWE. Besides, one more study was excluded due to insufficient data availability for calculating ORs and their variance [31]. And the characteristics and quality assessment of all included studies are summarized in Figure 1 and Table 1.
3.2. TLR-2 rs1898830 Polymorphism. The pooled ORs from the overall study with 2849 periodontitis patients and 2922 controls found a significant association between rs1898830 and chronic periodontitis risk under the allelic model (A allele vs. $\mathrm{G}$ allele: $p=0.014$, $\mathrm{OR}=1.208,95 \% \mathrm{CI}$ : 1.039 1.406), recessive model (GG vs. GA+AA: $p=0.028$, $\mathrm{OR}=$ $0.755,95 \%$ CI: $0.588-0.970$ ), and codominant model (GG VS. AA: $p=0.014$, OR $=0.681,95 \%$ CI: 0.501-0.925). In the subgroup analysis, the association was found only in Asian populations under the allelic model (A allele vs. $\mathrm{G}$ allele: $p$ $=0.037, \mathrm{OR}=1.186,95 \%$ CI: $1.010-1.394)$ and codominant model (GG VS. AA: $p=0.035$, OR $=0.704,95 \%$ CI: 0.508 $0.975)[18,19,25,29]$.The heterogeneity test for the pooled data sets was not significant $\left(I^{2}=0\right)$ for the TLR-2 rs1898830 polymorphism in five gene models, indicating the robustness of the meta-analysis for chronic periodontitis. Although a limited number of studies $(n=2)$ have been included for the rs 1898830 polymorphism, it suggested that rs1898830 $(A>G)$ may be associated with periodontitis risk in Asians (Figure 2 and Table 2).

3.3. TLR-2 rs3804100 Polymorphism. Seven studies with 2857 cases and 2452 controls did not report an association between periodontitis and TLR-2 rs3804100 polymorphism in five gene models ( $p=0.269$, OR $=0.922$, 95\% CI: 0.797-1.065). No association was found between the ethnic subgroup analysis and the disease type subgroup analysis. Subgroup analysis by disease type found that the marginal significance was in the codominant model of TC VS. TT ( $p=0.051, \mathrm{OR}=1.332,95 \%$ CI: 0.999-1.777); statistical heterogeneity varied from absent to moderate especially among Asians and $\mathrm{AgP}$ subgroup analysis $\left(I^{2}=66.9 \%\right.$ and $I^{2}=55.3 \%$, respectively). Moreover, the genotype distributions of all the included studies were in accordance with HWE except for the study of Ding et al. (Figure 3 and Table 3).

3.4. TLR-2 rs5734704 Polymorphism. Three studies derived from Caucasian ethnic involved 1596 cases and 1866 controls report the gene and showed that TLR-2 rs5734704 polymorphism might not contribute to periodontitis risk under all comparison models $(p=0.783$, OR $=0.966,95 \%$ CI: 0.755 1.237) either in Caucasian or disease study $(p=0.748, \mathrm{OR}=$ 1.053, 95\% CI: 0.770-1.439) (Figure 4 and Table 4).

3.5. TLR-2 rs5743708 Polymorphism. Thirteen studies with a total of 2361 cases and 2722 controls found no association between rs 5743708 and periodontitis risk under the five gene models ( $p=0.633$, OR $=0.949,95 \%$ CI: 0.765-1.177). Ethnic subgroup analyses showed that rs5743708 was associated with periodontitis risk in Asians under the allelic model ( $\mathrm{G}$ allele vs. A allele: $p=0.017, \mathrm{OR}=12.064,95 \%$ CI: $1.570-92.688)$, dominant model (GA+AA vs.GG: $p=0.016, \mathrm{OR}=0.08,95 \% \mathrm{CI}$ : $0.010-0.620$ ), and codominant model (GA VS. GG: $p=0.016$ , $\mathrm{OR}=0.080,95 \%$ CI: 0.821-1.282). Stratification by disease type indicated no association between the TLR-2 rs5743708 polymorphism and periodontitis in $\mathrm{CP}$ and $\mathrm{AgP}$. The overall genotyping distribution in all studies was in HWE equilibrium $[18-21,23,24,27-30]$ (Figure 5 and Table 5). 


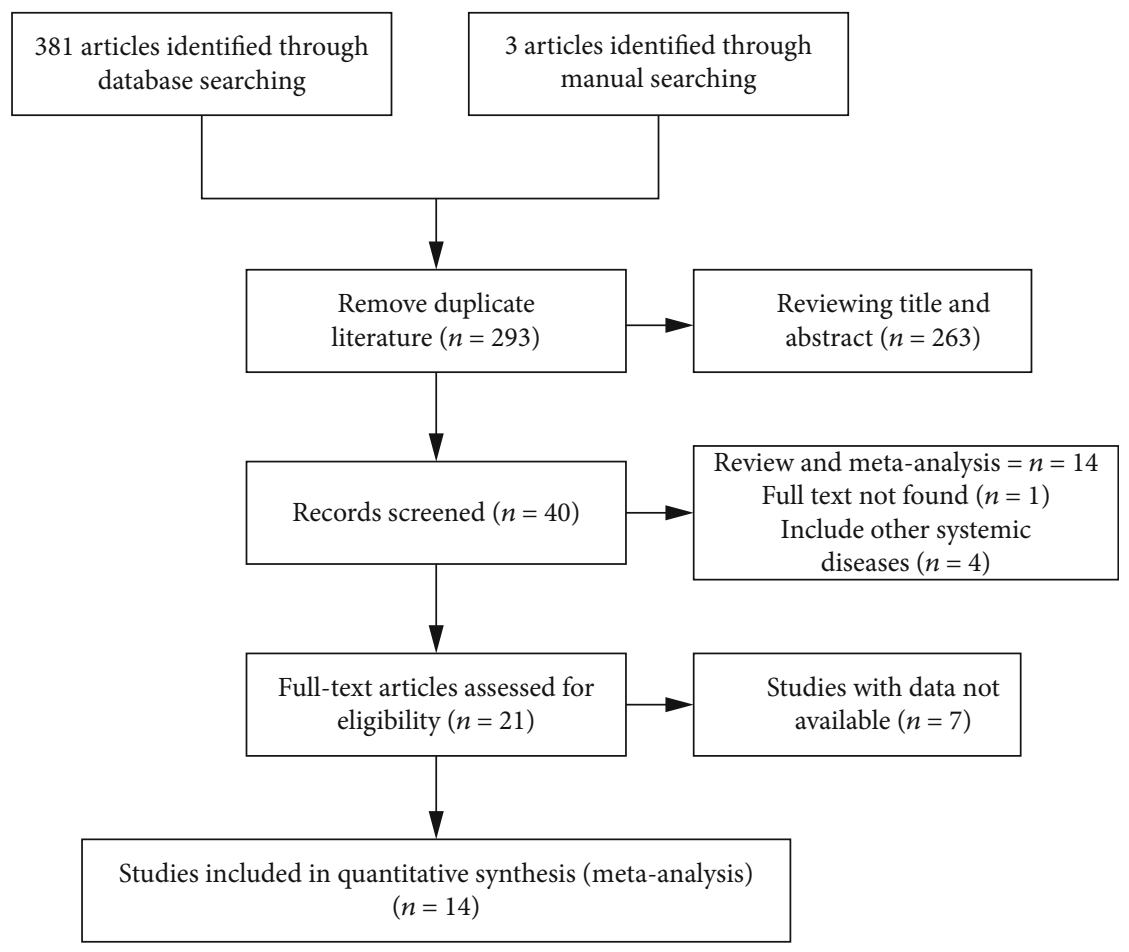

FIgURE 1: Flow diagram of the systematic review process.

3.6. TLR-2 rs13150331 Polymorphism. Only two studies research Asian populations and aggressive periodontitis about TLR-2 rs13150331 polymorphism and failed to find a significant association of this variant with the risk of AgP in Asian under all comparison models $[25,29](p=0.161$, OR $=1.121$, 95\% CI: 0.955-1.316) (Table 6).

3.7. Other TLR-2 Polymorphism. One SNP, namely, rs12191786, has been reported in two studies, but both of them deviated from the HWE equilibrium; therefore, we excluded the SNPs from our meta-analysis [28, 30](data not shown). And other SNPs rs7696323 in the study by Takahashi et al. have been investigated [26], and the association was found between the rs7696323 polymorphism and periodontitis susceptibility $(\mathrm{OR}=0.48, p=0.038)$ in Asians. In Fukusaki et al.'s study, they reported three point mutations in the $5^{\prime}$-untranslated region and one synonymous mutation in the coding region of TLR2 that were identified at basepair positions $-183,-148,-146$, and +2343 (corresponding to rs5743709), rs1816702, rs11938228, rs3804099 [22], and rs7656411 were reported in Richer et al.'s study [19], but they failed to find a significant association of this variant with the risk of CP in Asian populations.

3.8. Publication Bias Analysis. The Begg-Mazumdar test and the modified Egger test for funnel plot asymmetry for all study groups did not indicate any evidence of publication bias for TLR-2 [32, 33] (Figure 6).

\section{Discussion}

This is the first meta-analysis which comprehensively performed to investigate the relationship between TLR-2 poly- morphism and the risk of CP/AgP. We combined published data to assess the genetic association between the most commonly studied polymorphisms in the TLR-2 gene, including 18 studies with 3873 patients. The results showed that the rs1898830 $(A>G)$ was at a decreased risk of chronic periodontitis in Asians under the allelic model (A VS. G) and codominant model (GG VS. AA). Four papers studying rs1898830 showed that the polymorphism of this gene was not significantly correlated with the periodontitis susceptibility $[18,19,25,29]$, which was inconsistent with the results of our meta-analysis. In Richer et al., the gene distribution of the control group in the study of this locus of gene rs1898830 did not conform to the HWE, so we did not include Richer data in the analysis of this gene polymorphism. On the other hand, these studies were largely unable to draw definitive conclusions from their findings, even if their results were negative. Therefore, it is reasonable to think that the allele mutation of this $\operatorname{rs} 1898830(A>G)$ may be a protective factor for CP.

There are more than 20 single nucleotide polymorphism (SNP) sites for the TLR2 gene included in the Hapmap database. Exon 3 is the largest exon in TLR2 and contains several synonymous and nonsynonymous SNPS, as shown in the NCBI (NCBI build GRCh37).We identified four known SNPs: two common variants (rs574304, rs5743708 (Arg753Gln)) and two rare variants (rs3804099, rs3804100), a missense mutation in the third exon of the TLR2 gene, which has been the most internationally reported to be associated with periodontitis. In total, ten publications have examined the role of TLR-2 rs5743708 genetic variation in periodontitis, suggesting that rs5743708 may not be related to the periodontal disease, which is consistent in previous papers [34, 35]. While ethnic subgroup analyses demonstrated that TLR-2 


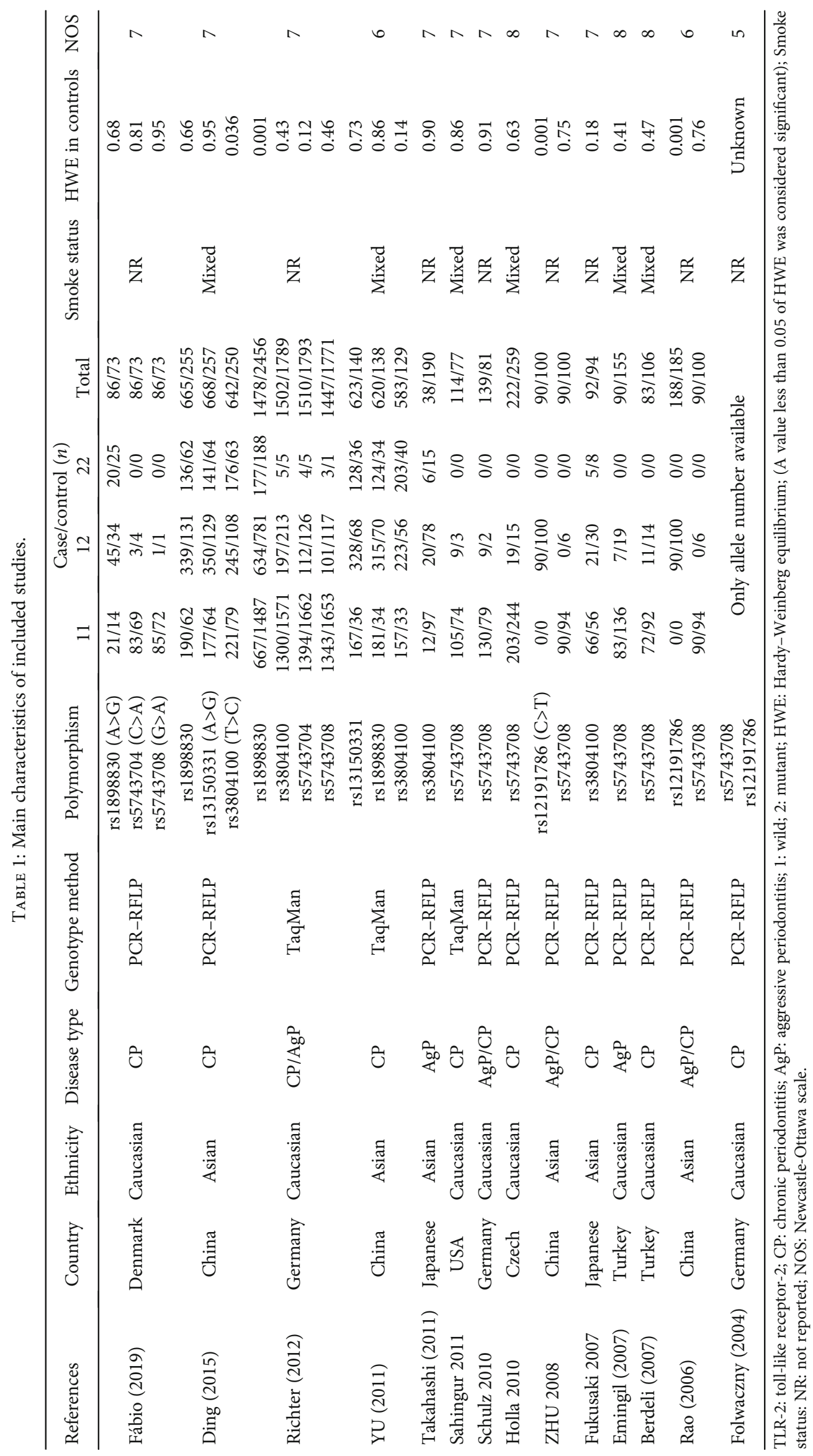




\begin{tabular}{|c|c|c|}
\hline $\begin{array}{l}\text { Study } \\
\text { ID }\end{array}$ & OR (95\% CI) & $\begin{array}{c}\% \\
\text { weight }\end{array}$ \\
\hline Fábio (2019) & $1.39(0.89,2.16)$ & 10.87 \\
\hline Ding (2015) & $1.18(0.96,1.44)$ & 55.53 \\
\hline YU (2011) & $1.20(0.93,1.56)$ & 33.61 \\
\hline Overall $(I$-squared $=0.0 \%, p=0.804)$ & $1.21(1.04,1.41)$ & 100.00 \\
\hline .463 & 2.16 & \\
\hline
\end{tabular}

(a)

\begin{tabular}{|c|c|c|}
\hline $\begin{array}{l}\text { Study } \\
\text { ID }\end{array}$ & $\mathrm{OR}(95 \% \mathrm{CI})$ & $\begin{array}{c}\% \\
\text { weight }\end{array}$ \\
\hline Fábio (2019) & $0.73(0.34,1.57)$ & 10.75 \\
\hline Ding (2015) & $0.80(0.58,1.12)$ & 54.99 \\
\hline YU (2011) & $0.79(0.52,1.21)$ & 34.26 \\
\hline Overall (I-squared $=0.0 \%, p=0.978$ & $0.79(0.62,1.01)$ & 100.00 \\
\hline .343 & 2.92 & \\
\hline
\end{tabular}

(b)

Figure 2: Forest plot of published case-control association studies of rs1898830 (A>G) in overall analysis fulfilling HWE. (a) Allele comparison (A allele vs. G allele). (b) Recessive model (GG vs. GA+AA).

TABLE 2: Main outcomes of the analyses on periodontitis and TLR-2 rs1898830 (A>G) polymorphism.

\begin{tabular}{|c|c|c|c|c|c|c|c|c|}
\hline Model & Subgroup & No. of studies & Case/control & OR & $95 \% \mathrm{CI}$ & $I^{2}$ & Pooling model & $p$ value \\
\hline \multirow{4}{*}{ A vs. $G$} & Overall (fulfill HWE) & 3 & $1371 / 466$ & 1.208 & $1.039-1.406$ & 0 & Fixed & 0.014 \\
\hline & Caucasian & 1 & $86 / 73$ & 1.387 & $0.890-2.162$ & 0 & Fixed & 0.149 \\
\hline & Asian & 2 & $1285 / 393$ & 1.186 & $1.010-1.394$ & 0 & Fixed & 0.037 \\
\hline & $\mathrm{CP}$ & 3 & $1371 / 466$ & 1.208 & $1.039-1.406$ & 0 & Fixed & 0.014 \\
\hline \multirow{4}{*}{ AG+GG vs. AA } & Overall (fulfill HWE) & 3 & $1371 / 466$ & 0.792 & $0.619-1.015$ & 0 & Fixed & 0.065 \\
\hline & Caucasian & 1 & $86 / 73$ & 0.734 & $0.343-1.575$ & 0 & Fixed & 0.428 \\
\hline & Asian & 2 & $1285 / 393$ & 0.799 & $0.615-1.038$ & 0 & Fixed & 0.093 \\
\hline & $\mathrm{CP}$ & 3 & $1371 / 466$ & 0.792 & 0.619-1.015 & 0 & Fixed & 0.065 \\
\hline \multirow{4}{*}{ GG vs. AG+AA } & Overall (fulfill HWE) & 3 & $1371 / 466$ & 0.755 & $0.588-0.970$ & 0 & Fixed & 0.028 \\
\hline & Caucasian & 1 & $86 / 73$ & 0.582 & $0.290-1.167$ & 0 & Fixed & 0.127 \\
\hline & Asian & 2 & $1285 / 393$ & 0.787 & $0.601-1.029$ & 0 & Fixed & 0.080 \\
\hline & $\mathrm{CP}$ & 3 & $1371 / 466$ & 0.755 & $0.588-0.970$ & 0 & Fixed & 0.028 \\
\hline \multirow{4}{*}{ AG vs. AA } & Overall (fulfill HWE) & 3 & $1371 / 466$ & 0.849 & $0.653-1.102$ & 0 & Fixed & 0.218 \\
\hline & Caucasian & 1 & $86 / 73$ & 0.882 & $0.393-1.983$ & 0 & Fixed & 0.762 \\
\hline & Asian & 2 & $1285 / 393$ & 0.845 & $0.641-1.113$ & 0 & Fixed & 0.231 \\
\hline & $\mathrm{CP}$ & 3 & $1371 / 466$ & 0.849 & $0.653-1.102$ & 0 & Fixed & 0.218 \\
\hline \multirow{4}{*}{ GG vs. AA } & Overall (fulfill HWE) & 3 & $1371 / 466$ & 0.681 & $0.501-0.925$ & 0 & Fixed & 0.014 \\
\hline & Caucasian & 1 & $86 / 73$ & 0.533 & $0.218-1.307$ & 0 & Fixed & 0.169 \\
\hline & Asian & 2 & $1285 / 393$ & 0.704 & $0.508-0.975$ & 0 & Fixed & 0.035 \\
\hline & $\mathrm{CP}$ & 3 & $1371 / 466$ & 0.681 & $0.501-0.925$ & 0 & Fixed & 0.014 \\
\hline
\end{tabular}

rs5743708 (G>A) was associated with Asian population under the allelic model ( $G$ allele vs. A allele) and dominant model (GA+AA vs.GG). This may be due to racial differences in genetic background. However, most previous studies have found that the occurrence of mutant genotypes/alleles is very rare. For example, Zhu et al. found only $6 \%$ heterozygotes in the control population in their study [28], so the study may be underpowered in this respect. There was no significant association between TLR-2 rs3804100 ( $\mathrm{T}>\mathrm{C}$ ) among the overall and subgroup analysis. We found the marginal significance only in the codominant model on subgroup analysis in AgP. Among five studies related to the TLR-2 rs3804100 (T>C) and periodontitis, there was only one study conducted by Takahashi et al. which suggested that the rs3804100 (T>C) gene polymorphism in Asian subjects was associated with AgP [26]. Only two articles reported an association between this site and AgP in Asians. We could not conclude the significant result due to the small sample size. Additionally, no association was identified between the other TLR-2 polymorphism and periodontitis risk.

No significant association was observed for other sites mentioned in the papers between genetic TLR-2 polymorphisms of rs13150331 (A>G), rs12191786 (C>T), rs1816702 $(\mathrm{C}>\mathrm{T}), \quad r s 11938228(\mathrm{C}>\mathrm{A}), \quad r s 3804099(\mathrm{~T}>\mathrm{C}), \quad$ rs7656411 $(\mathrm{T}>\mathrm{G})$, and base-pair positions $-183(\mathrm{~A}>\mathrm{G}),-148(\mathrm{C}>\mathrm{T}),-146$ $(\mathrm{T}>\mathrm{G})$, and $+2343(\mathrm{G}>\mathrm{A})$ (corresponding to rs5743709), except for one study, which reported decreased risk of rs7696323 polymorphism $(\mathrm{C}>\mathrm{T})$ in aggressive periodontitis 


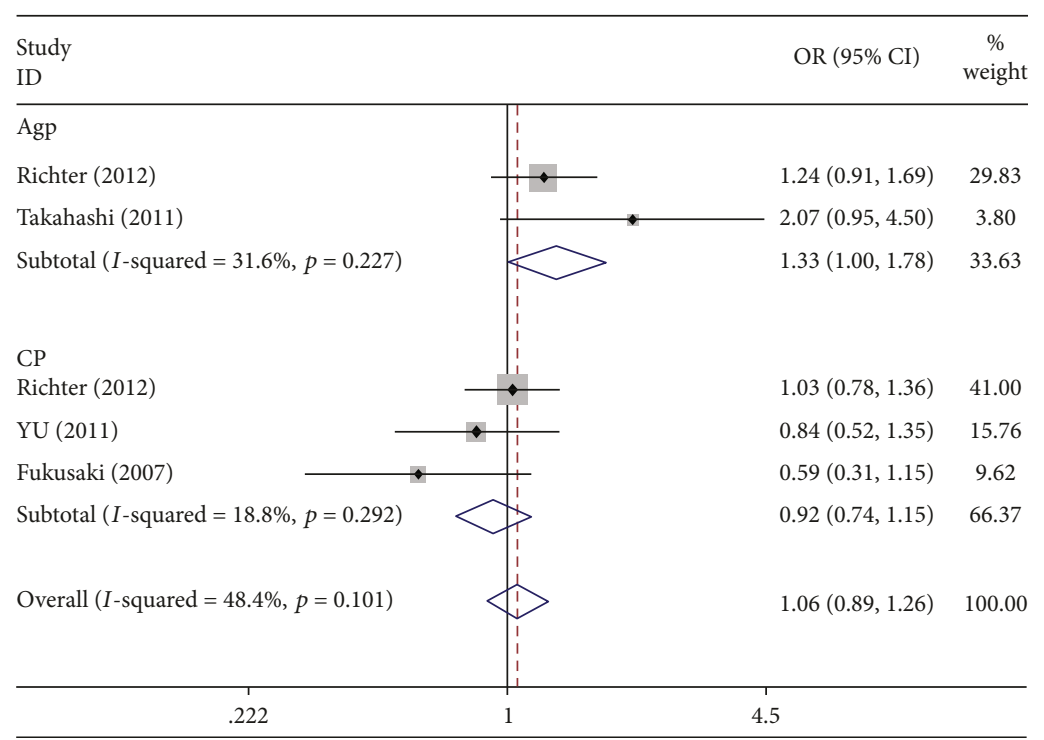

Figure 3: Forest plot of published case-control association studies of rs3804100 (T>C) in disease subgroup analysis fulfilling HWE. Codominant model (TC VS. TT).

TABLE 3: Main outcomes of the analyses on periodontitis and TLR-2 rs3804100 (T>C) polymorphism.

\begin{tabular}{|c|c|c|c|c|c|c|c|c|}
\hline Model & Subgroup & No. of studies & Case/control & OR & $95 \% \mathrm{CI}$ & $I^{2}$ & Pooling model & $p$ \\
\hline \multirow{5}{*}{ T vs. C } & Overall (fulfill HWE) & 4 & $2215 / 2202$ & 0.922 & $0.797-1.065$ & $65.6 \%$ & Random & 0.269 \\
\hline & Caucasian & 1 & $1502 / 1789$ & 0.898 & $0.738-1.092$ & 0 & Fixed & 0.282 \\
\hline & Asian & 3 & $713 / 413$ & 0.951 & $0.766-1.179$ & $76.7 \%$ & Random & 0.645 \\
\hline & $\mathrm{CP}$ & 3 & $1580 / 1302$ & 1.012 & $0.849-1.207$ & $43.4 \%$ & Fixed & 0.893 \\
\hline & $\mathrm{AgP}$ & 2 & $635 / 900$ & 0.713 & $0.474-1.072$ & $51.5 \%$ & Random & 0.104 \\
\hline \multirow{5}{*}{$\mathrm{TC}+\mathrm{CC}$ vs. TT } & Overall (fulfill HWE) & 4 & $2215 / 2202$ & 1.047 & $0.718-1.528$ & $63.9 \%$ & Random & 0.812 \\
\hline & Caucasian & 1 & $1502 / 1789$ & 1.120 & $0.912-1.375$ & 0 & Fixed & 0.280 \\
\hline & Asian & 3 & $713 / 413$ & 1.033 & $0.530-2.014$ & $74.1 \%$ & Random & 0.924 \\
\hline & $\mathrm{CP}$ & 3 & $1580 / 1302$ & 0.942 & $0.758-1.170$ & $31.8 \%$ & Fixed & 0.589 \\
\hline & $\mathrm{AgP}$ & 2 & $635 / 900$ & 1.511 & $0.854-2.672$ & $55.3 \%$ & Random & 0.156 \\
\hline \multirow{5}{*}{$\mathrm{CC}$ vs. TC+TT } & Overall (fulfill HWE) & 4 & $2215 / 2202$ & 1.188 & $0.839-1.681$ & 0 & Fixed & 0.332 \\
\hline & Caucasian & 1 & $1502 / 1789$ & 1.192 & $0.344-4.124$ & 0 & Fixed & 0.782 \\
\hline & Asian & 3 & $713 / 413$ & 1.187 & $0.827-1.705$ & $23.3 \%$ & Fixed & 0.352 \\
\hline & $\mathrm{CP}$ & 3 & $1580 / 1302$ & 1.129 & $0.778-1.637$ & 0 & Fixed & 0.523 \\
\hline & $\mathrm{AgP}$ & 2 & $635 / 900$ & 1.706 & $0.667-4.363$ & 0 & Fixed & 0.265 \\
\hline \multirow{5}{*}{ TC vs. TT } & Overall (fulfill HWE) & 4 & $2215 / 2202$ & 1.012 & $0.700-1.463$ & $57.2 \%$ & Random & 0.948 \\
\hline & Caucasian & 1 & $1502 / 1789$ & 1.118 & $0.909-1.375$ & 0 & Fixed & 0.293 \\
\hline & Asian & 3 & $713 / 413$ & 0.918 & $0.653-1.290$ & $66.9 \%$ & Random & 0.621 \\
\hline & $\mathrm{CP}$ & 3 & $1580 / 1302$ & 0.921 & $0.735-1.154$ & $18.8 \%$ & Fixed & 0.474 \\
\hline & $\mathrm{AgP}$ & 2 & $635 / 900$ & 1.332 & $0.999-1.777$ & $31.6 \%$ & Fixed & $0.051^{*}$ \\
\hline \multirow{5}{*}{ CC vs. TT } & Overall (fulfill HWE) & 4 & $2215 / 2202$ & 1.122 & $0.749-1.681$ & $40.6 \%$ & Fixed & 0.577 \\
\hline & Caucasian & 1 & $1502 / 1789$ & 1.208 & $0.349-4.183$ & 0 & Fixed & 0.765 \\
\hline & Asian & 3 & $713 / 413$ & 1.112 & $0.725-1.706$ & $60.3 \%$ & Random & 0.626 \\
\hline & $\mathrm{CP}$ & 3 & $1580 / 1302$ & 0.997 & $0.641-1.550$ & 0 & Fixed & 0.990 \\
\hline & $\mathrm{AgP}$ & 2 & $635 / 900$ & 2.203 & $0.806-6.023$ & $35.1 \%$ & Fixed & 0.124 \\
\hline
\end{tabular}

in Asians [26]. As for rs12191786, three studies failed to identify the reported mutations rs 12191786 in TLR-2. Fukusaki et al. performed direct sequencing of TLR 2 and identified five base-pair positions $-183,-148,-146,+1350$ (corresponding to rs3804100) and +2343 (corresponding to rs5743709). Based on the associations observed in our meta-analysis, we 


\begin{tabular}{|c|c|c|}
\hline $\begin{array}{l}\text { Study } \\
\text { ID }\end{array}$ & OR $(95 \%$ CI $)$ & $\begin{array}{c}\% \\
\text { weight }\end{array}$ \\
\hline Fábio (2019) & $1.59(0.35,7.21)$ & 2.09 \\
\hline Richter (2012) & $0.95(0.74,1.22)$ & 97.91 \\
\hline Overall $(I$-squared $=0.0 \%, p=0.514)$ & $0.97(0.75,1.24)$ & 100.00 \\
\hline .139 & 7.21 & \\
\hline
\end{tabular}

Figure 4: Forest plot of published case-control association studies of rs5734704 (C>A) in overall analysis fulfilling HWE. Allele comparison model (A allele vs. G allele).

TABLE 4: Main outcomes of the analyses on periodontitis and TLR-2 rs5743704 (C>A) polymorphism.

\begin{tabular}{|c|c|c|c|c|c|c|c|c|}
\hline Model & Subgroup & No. of studies & Case/control & OR & $95 \% \mathrm{CI}$ & $I^{2}$ & Pooling model & $p$ \\
\hline \multirow{4}{*}{ C vs. A } & Overall (fulfill HWE) & 2 & $1596 / 1866$ & 0.966 & $0.755-1.237$ & 0 & Fixed & 0.783 \\
\hline & Caucasian & 2 & $1596 / 1866$ & 0.966 & $0.755-1.237$ & 0 & Fixed & 0.783 \\
\hline & $\mathrm{CP}$ & 2 & $998 / 1156$ & 1.053 & $0.770-1.439$ & 0 & Fixed & 0.748 \\
\hline & $\mathrm{AgP}$ & 1 & $598 / 710$ & 0.837 & $0.559-1.253$ & 0 & Fixed & 0.386 \\
\hline \multirow{4}{*}{$\mathrm{CA}+\mathrm{AA}$ vs. $\mathrm{CC}$} & Overall (fulfill HWE) & 2 & $1596 / 1866$ & 1.040 & $0.805-1.344$ & 0 & Fixed & 0.764 \\
\hline & Caucasian & 2 & $1596 / 1866$ & 1.040 & $0.805-1.344$ & 0 & Fixed & 0.764 \\
\hline & $\mathrm{CP}$ & 2 & $998 / 1156$ & 0.954 & $0.690-1.319$ & 0 & Fixed & 0.776 \\
\hline & $\mathrm{AgP}$ & 1 & $598 / 710$ & 1.203 & $0.791-1.831$ & 0 & Fixed & 0.388 \\
\hline \multirow{4}{*}{$\mathrm{AA}$ vs. $\mathrm{CA}+\mathrm{CC}$} & Overall (fulfill HWE) & 2 & $1596 / 1866$ & 0.950 & $0.255-3.543$ & 0 & Fixed & 0.939 \\
\hline & Caucasian & 2 & $1596 / 1866$ & 0.950 & $0.255-3.543$ & 0 & Fixed & 0.939 \\
\hline & $\mathrm{CP}$ & 2 & $998 / 1156$ & 0.791 & $0.132-4.475$ & 0 & Fixed & 0.798 \\
\hline & $\mathrm{AgP}$ & 1 & $598 / 710$ & 1.188 & $0.167-8.459$ & 0 & Fixed & 0.863 \\
\hline \multirow{4}{*}{ CA vs. CC } & Overall (fulfill HWE) & 2 & $1596 / 1866$ & 1.043 & $0.804-1.354$ & 0 & Fixed & 0.749 \\
\hline & Caucasian & 2 & $1596 / 1866$ & 1.043 & $0.804-1.354$ & 0 & Fixed & 0.749 \\
\hline & $\mathrm{CP}$ & 2 & $998 / 1156$ & 0.960 & $0.691-1.333$ & 0 & Fixed & 0.806 \\
\hline & $\mathrm{AgP}$ & 1 & $598 / 710$ & 1.203 & $0.784-1.847$ & 0 & Fixed & 0.397 \\
\hline \multirow{4}{*}{ AA vs. CC } & Overall (fulfill HWE) & 2 & $1596 / 1866$ & 0.954 & $0.256-3.559$ & 0 & Fixed & 0.944 \\
\hline & Caucasian & 2 & $1596 / 1866$ & 0.954 & $0.256-3.559$ & 0 & Fixed & 0.944 \\
\hline & $\mathrm{CP}$ & 2 & $998 / 1156$ & 0.790 & $0.132-4.739$ & 0 & Fixed & 0.797 \\
\hline & AgP & 1 & $598 / 710$ & 1.203 & $0.169-8.570$ & 0 & Fixed & 0.853 \\
\hline
\end{tabular}

concluded that most of these SNPs are only reported in one study and the mutant allele frequency between case and control was too small to have further analysis, because of the small sample size and the low frequency of alleles. Thus, the conclusions of the association between these SNPs and periodontitis are questionable.

In recent years, increasingly more studies on TLR single nucleotide polymorphisms (SNPs) have been reported. The main consideration factor is that TLR SNPs may reduce the response efficiency of TLR ligands, thus affecting the susceptibility to infectious diseases [36, 37]. Aggressive periodontitis and chronic periodontitis are complex diseases with multifactor etiology, genotyping a single genetic variation that often fails to draw conclusions about whether the genes in question are related to the disease. So, we identified several SNPs which had directly been investigated for its potential association with periodontitis in previous studies. Recently, some studies have shifted the focus to genome-wide approaches rather than candidate gene approaches, thereby considering the possibility that multiple genes may be causal factors in periodontitis. Schaefer et al. performed a genomewide association study in two independent phases and found that the GLT6D1 gene was not significantly expressed in healthy tissues and gingival inflammatory tissues [38]. Thus, periodontitis appears to be associated with several sites, each of which has a relatively small effect. Only a few studies have 


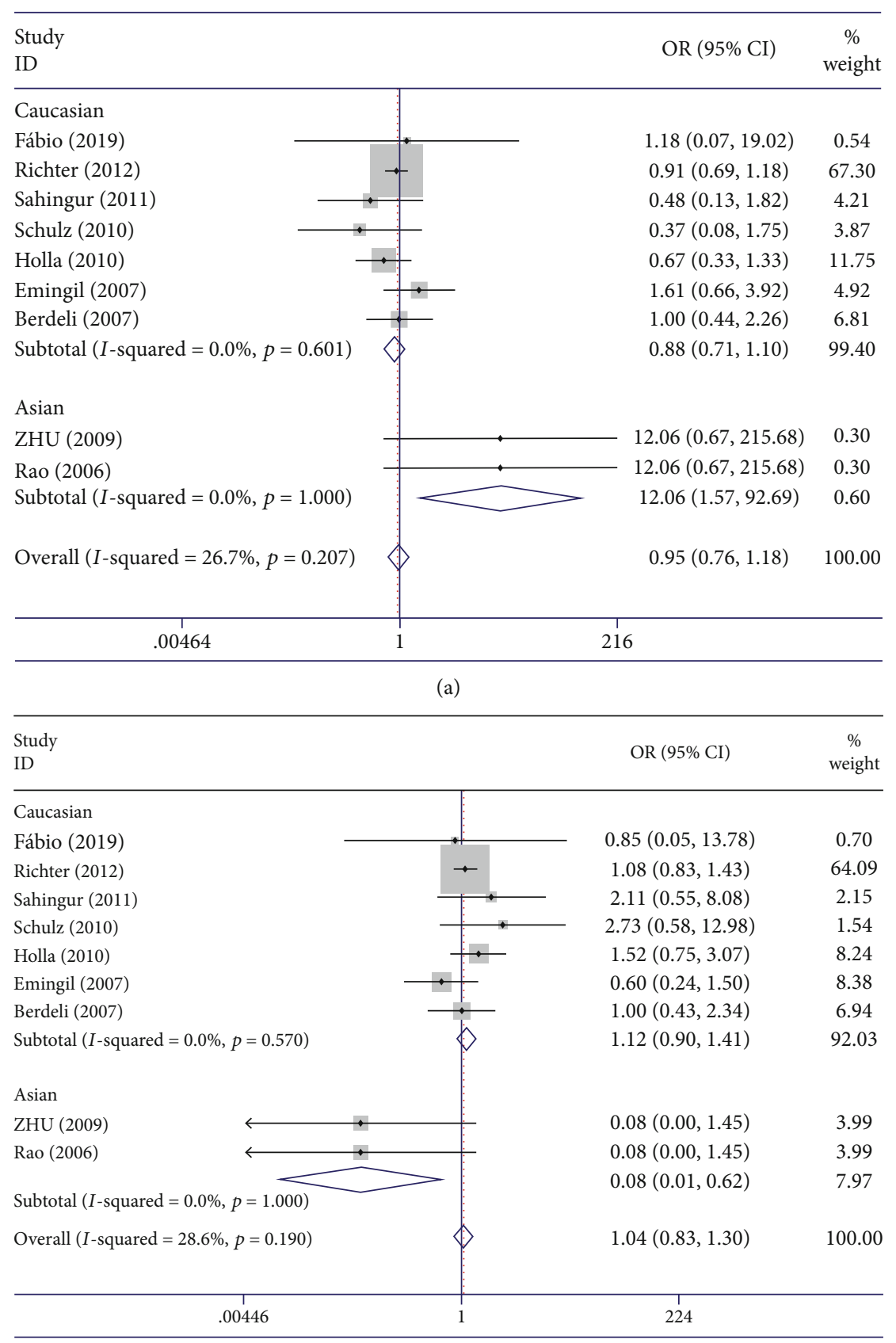

(b)

Figure 5: Forest plot of published case-control association studies of rs5743708 (G>A) in ethnic subgroup analysis fulfilling HWE. (a) allele comparison model (G allele vs. A allele) (b) dominant model (GA+AA vs.GG).

investigated the cooccurrence of multiple polymorphisms, although more research may be forthcoming.

So far, a number of studies have evaluated the association between PRRs, SNPs, and periodontitis, including CD14, TLR2, TLR4, and MBL2. However, most of the studies on gene polymorphism of TLR-2 have considered that there is no obvious correlation between TLR-2 polymorphism and periodontitis. Therefore, the meta-analysis on the relationship between TLR-2 gene polymorphism and periodontitis susceptibility has not been reported alone, while the metaanalysis on TLR-4 and CD14 has been published; the conclusions are still inconsistent $[34,35,39,40]$. Our meta-analysis was the first to independently analyze the relationship between TLR-2 and periodontitis, including 18 studies and six common SNPs and some unusual SNPs. We also found that the rs1898830 $(A>G)$ may be a protective factor for periodontitis susceptibility, which has not been found in previous studies. In particualr, the rest of our results are consistent with previous studies. Although unlikely, rare disease-related variants may exist in these exons, which were not identified in our study. Currently, the availability of genome-wide arrays allows a wide range of genes to be scanned and identified in relation to disease processes. Although these methods were not used in these included 
TABLE 5: Main outcomes of the analyses on periodontitis and TLR-2 rs5743708 (G>A) polymorphism.

\begin{tabular}{|c|c|c|c|c|c|c|c|c|}
\hline Model & Subgroup & No. of studies & Case/control & OR & $95 \% \mathrm{CI}$ & $I^{2}$ & Pooling model & $p$ \\
\hline \multirow{5}{*}{ G vs. A } & Overall (fulfill HWE) & 9 & $2361 / 2722$ & 0.949 & $0.765-1.177$ & $26.7 \%$ & Fixed & 0.633 \\
\hline & Caucasian & 7 & $2181 / 2522$ & 0.881 & 0.707-1.099 & 0 & Fixed & 0.262 \\
\hline & Asian & 2 & $180 / 200$ & 12.064 & $1.570-92.688$ & 0 & Fixed & $0.017^{*}$ \\
\hline & $\mathrm{CP}$ & 8 & $1520 / 1823$ & 0.845 & $0.646-1.104$ & 0 & Fixed & 0.216 \\
\hline & AgP & 5 & $841 / 1080$ & 1.149 & $0.805-1.640$ & $17.7 \%$ & Fixed & 0.444 \\
\hline \multirow{5}{*}{ GA+AA vs. GG } & Overall (fulfill HWE) & 9 & $2361 / 2722$ & 1.041 & $0.834-1.298$ & $28.6 \%$ & Fixed & 0.724 \\
\hline & Caucasian & 7 & $2181 / 2522$ & 1.124 & 0.897-1.409 & 0 & Fixed & 0.311 \\
\hline & Asian & 2 & $180 / 200$ & 0.080 & $0.010-0.620$ & 0 & Fixed & $0.016^{*}$ \\
\hline & $\mathrm{CP}$ & 8 & $1520 / 1823$ & 1.179 & $0.896-1.551$ & 0 & Fixed & 0.241 \\
\hline & $\mathrm{AgP}$ & 5 & $841 / 1080$ & 0.849 & $0.590-1.221$ & $18.8 \%$ & Fixed & 0.377 \\
\hline \multirow{5}{*}{ AA vs. GA+GG } & Overall (fulfill HWE) & 9 & $2361 / 2722$ & 3.677 & $0.382-35.389$ & 0 & Fixed & 0.260 \\
\hline & Caucasian & 7 & $2181 / 2522$ & 3.677 & $0.382-35.389$ & 0 & Fixed & 0.260 \\
\hline & Asian & 2 & $180 / 200$ & - & - & - & Fixed & - \\
\hline & $\mathrm{CP}$ & 8 & $1520 / 1823$ & 2.529 & $0.229-27.935$ & 0 & Fixed & 0.449 \\
\hline & $\mathrm{AgP}$ & 5 & $841 / 1080$ & 2.865 & $0.422-19.464$ & 0 & Fixed & 0.442 \\
\hline \multirow{5}{*}{ GA vs. GG } & Overall (fulfill HWE) & 9 & $2361 / 2722$ & 1.026 & $0.821-1.282$ & 0 & Fixed & 0.820 \\
\hline & Caucasian & 7 & $2181 / 2522$ & 1.109 & $0.883-1.392$ & 0 & Fixed & 0.374 \\
\hline & Asian & 2 & $180 / 200$ & 0.080 & $0.821-1.282$ & 0 & Fixed & $0.016^{*}$ \\
\hline & $\mathrm{CP}$ & 8 & $1520 / 1823$ & 1.166 & $0.885-1.538$ & 0 & Fixed & 0.275 \\
\hline & $\mathrm{AgP}$ & 5 & $841 / 1080$ & 0.833 & $0.578-1.201$ & $17.5 \%$ & Fixed & 0.328 \\
\hline \multirow{5}{*}{ AA vs. GG } & Overall (fulfill HWE) & 9 & $2361 / 2722$ & 3.692 & $0.384-35.538$ & 0 & Fixed & 0.258 \\
\hline & Caucasian & 7 & $2181 / 2522$ & 3.692 & $0.384-35.538$ & 0 & Fixed & 0.258 \\
\hline & Asian & 2 & $180 / 200$ & - & - & - & Fixed & - \\
\hline & $\mathrm{CP}$ & 8 & $1520 / 1823$ & 2.555 & $0.231-28.229$ & 0 & Fixed & 0.444 \\
\hline & AgP & 5 & $841 / 1080$ & 2.878 & $0.424-19.560$ & 0 & Fixed & 0.444 \\
\hline
\end{tabular}

TABLE 6: Main outcomes of the analyses on periodontitis and TLR-2 rs13150331 polymorphism.

\begin{tabular}{|c|c|c|c|c|c|c|c|c|}
\hline Model & Subgroup & No. of studies & Case/control & OR & $95 \% \mathrm{CI}$ & $I^{2}$ & Pooling model & $p$ \\
\hline \multirow{3}{*}{ A vs.G } & Overall (fulfill HWE) & 2 & $1291 / 397$ & 1.121 & $0.955-1.316$ & 0 & Fixed & 0.161 \\
\hline & Asian & 2 & $1291 / 397$ & 1.121 & $0.955-1.316$ & 0 & Fixed & 0.161 \\
\hline & $\mathrm{CP}$ & 2 & $1291 / 397$ & 1.121 & $0.955-1.316$ & 0 & Fixed & 0.161 \\
\hline \multirow{3}{*}{ AG+GG vs. AA } & Overall (fulfill HWE) & 2 & $1291 / 397$ & 0.930 & $0.717-1.205$ & 0 & Fixed & 0.581 \\
\hline & Asian & 2 & $1291 / 397$ & 0.930 & $0.717-1.205$ & 0 & Fixed & 0.581 \\
\hline & $\mathrm{CP}$ & 2 & $1291 / 397$ & 0.930 & $0.717-1.205$ & 0 & Fixed & 0.581 \\
\hline \multirow{3}{*}{ GG vs. AG+AA } & Overall (fulfill HWE) & 2 & $1291 / 397$ & 0.783 & $0.601-1.021$ & 0 & Fixed & 0.071 \\
\hline & Asian & 2 & $1291 / 397$ & 0.783 & $0.601-1.021$ & 0 & Fixed & 0.071 \\
\hline & $\mathrm{AgP}$ & 2 & $1291 / 397$ & 0.783 & $0.601-1.021$ & 0 & Fixed & 0.071 \\
\hline \multirow{3}{*}{ AG vs. AA } & Overall (fulfill HWE) & 2 & $1291 / 397$ & 1.003 & $0.762-1.321$ & 0 & Fixed & 0.983 \\
\hline & Asian & 2 & $1291 / 397$ & 1.003 & $0.762-1.321$ & 0 & Fixed & 0.983 \\
\hline & $\mathrm{CP}$ & 2 & $1291 / 397$ & 1.003 & $0.762-1.321$ & 0 & Fixed & 0.983 \\
\hline \multirow{3}{*}{ GG vs. AA } & Overall (fulfill HWE) & 2 & $1291 / 397$ & 0.785 & $0.569-1.082$ & 0 & Fixed & 0.140 \\
\hline & Asian & 2 & $1291 / 397$ & 0.785 & $0.569-1.082$ & 0 & Fixed & 0.140 \\
\hline & $\mathrm{CP}$ & 2 & $1291 / 397$ & 0.785 & $0.569-1.082$ & 0 & Fixed & 0.140 \\
\hline
\end{tabular}

studies and only a limited number of genes were studied, our results provide promising evidence for designing future studies to increase the sample size to determine genetic susceptibility to periodontitis in PRRs.
Our study has some limitations. First, the included studies varied in several ways, including age, gender, and smoking status. Only six articles cited smoking habits as a confounding factor. And we did not provide subgroup analysis for 
Begg's funnel plot with pseudo 95\% confidence limits

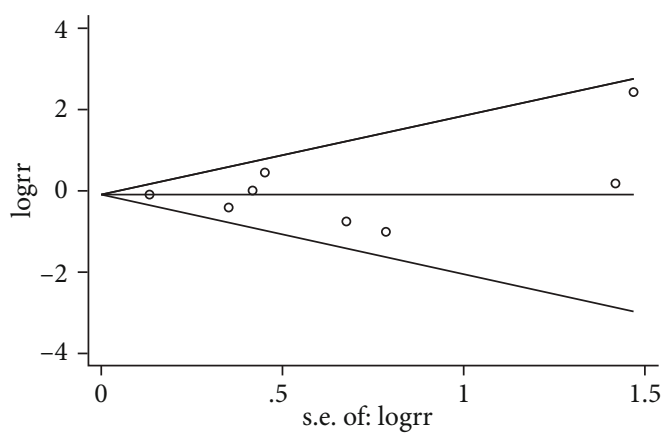

(a)

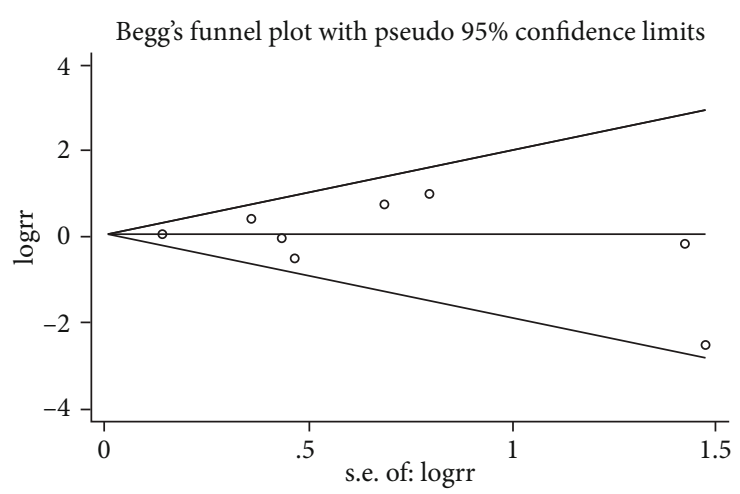

(b)

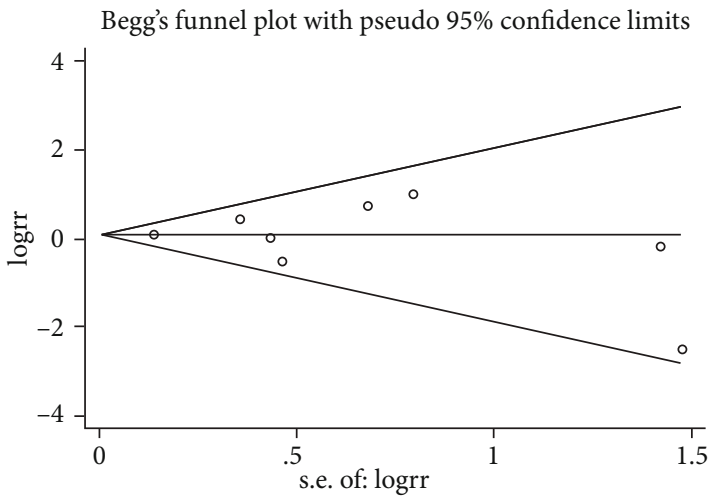

(c)

Figure 6: Begg's funnel plot of the TLR-2 rs5743708 (G>A) polymorphism and CP risk in different contrast models: (a) allele comparison model (G allele vs. A allele); (b) codominant model (GA vs. GG); and (c) dominant genetic model (AA+GA vs. GG).

smoke status. Periodontitis is a multifactorial disease, and due to the lack of appropriate proportions of eligible studies, it is not possible to make detailed assessments of potential interactions, as gene-gene, gene-environment, or even different polymorphism loci of the same gene may regulate the risk of periodontitis. Second, due to the extremely low allele frequency of some SNPs such as rs5743704 (C>A), rs5743708 $(\mathrm{G}>\mathrm{A})$, and $\mathrm{rs} 12191786(\mathrm{C}>\mathrm{T})$, there is often no homozygous mutation or even no heterozygosity. For these SNPs, the calculation of some gene models (dominant model and a recessive model) cannot be carried out, which probably will affect our analysis results. In our meta-analysis, many individual studies seem to be underpowered. A sample size of 5002000 case-control pairs is required to detect moderate genetic effect size [41]. And a large number of potential genetic risk factors and a relatively low frequency of polymorphisms may result in initial observations not being confirmed by subsequent studies. Although we have studied only a few SNPs of one gene, our results provide positive evidence for designing future studies to increase the sample size to determine genetic susceptibility to periodontitis in TLR.

\section{Conclusions}

In summary, this meta-analysis of published data shows that a protect correlation between the $\operatorname{rs} 1898830(A>G)$ and rs5743708 ( $\mathrm{G}>\mathrm{A})$ was associated with periodontitis risk in Asians. Limited by the number of included samples and
SNP sites in the current association study, the above conclusions need to be further confirmed by the large sample, multiethnics, and multi-SNP-sites studies in future. We consider that the genetic information of TLR2 site has a certain reference value for the diagnosis, prevention, and treatment of chronic periodontitis. If this genetic information is available, it may provide preventive strategies and therapeutic interventions for patients with high susceptibility.

\section{Data Availability}

All data generated or analyzed during this study are included in this article.

\section{Conflicts of Interest}

The authors declare no potential conflicts of interest with respect to the authorship and/or publication of this article.

\section{Acknowledgments}

The authors gratefully acknowledge the support of the National Natural Science Foundation of China (Grant No. 81760194), Abasijiang Aisaiti for providing genotypes data and valuable comments; and Wu Zhong Peng and Wang Ting Ting for their assistance in data collection. In addition, the authors specifically acknowledge the guidance and insightful ideas provided by Professor Zhao Jin. 


\section{References}

[1] M. L. Laine, S. Jepsen, and B. G. Loos, "Progress in the identification of genetic factors in periodontitis," Current Oral Health Reports, vol. 1, no. 4, pp. 272-278, 2014.

[2] B. L. Pihlstrom, B. S. Michalowicz, and N. W. Johnson, "Periodontal diseases," The Lancet, vol. 366, no. 9499, pp. 18091820, 2005.

[3] S. Listl, J. Galloway, P. A. Mossey, and W. Marcenes, "Global economic impact of dental diseases," Journal of Dental Research, vol. 94, no. 10, pp. 1355-1361, 2015.

[4] L. N. Borrell and P. N. Papapanou, "Analytical epidemiology of periodontitis," Journal of Clinical Periodontology, vol. 32, Supplement 6, pp. 132-158, 2005.

[5] R. P. Darveau, "Periodontitis: a polymicrobial disruption of host homeostasis," Microbiology, vol. 8, no. 7, pp. 481-490, 2010.

[6] B. S. Michalowicz, S. R. Diehl, J. C. Gunsolley et al., "Evidence of a substantial genetic basis for risk of adult periodontitis," Journal of Periodontology, vol. 71, no. 11, pp. 1699-1707, 2000.

[7] D. F. Kinane, P. G. Stathopoulou, and P. N. Papapanou, "Periodontal diseases," Nature Reviews Disease Primers, vol. 3, no. 1, article 17038, 2017.

[8] A. Teumer, B. Holtfreter, U. Volker et al., "Genome-wide association study of chronic periodontitis in a general German population," Journal of Clinical Periodontology, vol. 40, no. 11, pp. 977-985, 2013.

[9] K. Divaris, K. L. Monda, K. E. North et al., "Exploring the genetic basis of chronic periodontitis: a genome-wide association study," Human Molecular Genetics, vol. 22, no. 11, pp. 2312-2324, 2013.

[10] S. Freitag-Wolf, H. Dommisch, C. Graetz et al., "Genome-wide exploration identifies sex-specific genetic effects of alleles upstream NPY to increase the risk of severe periodontitis in men," Journal of Clinical Periodontology, vol. 41, no. 12, pp. 1115-1121, 2014.

[11] M. Fukata and M. Arditi, "The role of pattern recognition receptors in intestinal inflammation," Mucosal Immunology, vol. 6, no. 3, pp. 451-463, 2013.

[12] M. Estruch, C. Bancells, L. Beloki, J. L. Sanchez-Quesada, J. Ordonez-Llanos, and S. Benitez, "CD14 and TLR4 mediate cytokine release promoted by electronegative LDL in monocytes," Atherosclerosis, vol. 229, no. 2, pp. 356-362, 2013.

[13] Y. Mori, A. Yoshimura, T. Ukai, E. Lien, T. Espevik, and Y. Hara, "Immunohistochemical localization of Toll-like receptors 2 and 4 in gingival tissue from patients with periodontitis," Oral Microbiology and Immunology, vol. 18, no. 1, pp. 54-58, 2003.

[14] V. Gelani, A. P. Fernandes, T. H. Gasparoto et al., "The role of toll-like receptor 2 in the recognition of Aggregatibacter actinomycetemcomitans," Journal of Periodontology, vol. 80, no. 12, pp. 2010-2019, 2009.

[15] N. Scheres, M. L. Laine, P. M. Sipos et al., "Periodontal ligament and gingival fibroblasts from periodontitis patients are more active in interaction with Porphyromonas gingivalis," Journal of Periodontal Research, vol. 46, no. 4, pp. 407-416, 2011.

[16] M. L. Laine, B. G. Loos, and W. Crielaard, "Gene polymorphisms in chronic periodontitis," International Journal of Dentistry, vol. 2010, Article ID 324719, 22 pages, 2010.

[17] D. Moher, A. Liberati, J. Tetzlaff, D. G. Altman, and for the PRISMA Group, "Preferred reporting items for systematic reviews and meta-analyses: the PRISMA statement," $B M J$, vol. 339, article b2535, 2009.

[18] F. R. M. Leite, C. Enevold, K. Bendtzen, V. Baelum, and R. López, "Pattern recognition receptor polymorphisms in early periodontitis," Journal of Periodontology, vol. 90, no. 6, pp. 647-654, 2018.

[19] G. M. Richter, C. Graetz, P. Pohler et al., "Common genetic risk variants of TLR2 are not associated with periodontitis in large European case-control populations," Journal of Clinical Periodontology, vol. 39, no. 4, pp. 315-322, 2012.

[20] S. E. Sahingur, X. J. Xia, J. Gunsolley, H. A. Schenkein, R. J. Genco, and E. De Nardin, "Single nucleotide polymorphisms of pattern recognition receptors and chronic periodontitis," Journal of Periodontal Research, vol. 46, no. 2, pp. 184-192, 2011.

[21] L. I. Holla, J. Vokurka, B. Hrdlickova, P. Augustin, and A. Fassmann, "Association of Toll-like receptor 9 haplotypes with chronic periodontitis in Czech population," Journal of Clinical Periodontology, vol. 37, no. 2, pp. 152-159, 2010.

[22] T. Fukusaki, N. Ohara, Y. Hara, A. Yoshimura, and K. Yoshiura, "Evidence for association between a Toll-like receptor 4 gene polymorphism and moderate/severe periodontitis in the Japanese population," Journal of Periodontal Research, vol. 42, no. 6, pp. 541-545, 2007.

[23] G. Emingil, A. Berdeli, H. Baylas et al., "Toll-like receptor 2 and 4 gene polymorphisms in generalized aggressive periodontitis," Journal of Periodontology, vol. 78, no. 10, pp. 1968-1977, 2007.

[24] A. Berdeli, G. Emingil, B. Han Saygan et al., “TLR2 Arg753Gly, TLR4 Asp299Gly and Thr399Ile gene polymorphisms are not associated with chronic periodontitis in a Turkish population," Journal of Clinical Periodontology, vol. 34, no. 7, pp. 551-557, 2007.

[25] Y. S. Ding, Y. Zhao, Y. Y. Xiao, and G. Zhao, “Toll-like receptor 4 gene polymorphism is associated with chronic periodontitis," International Journal of Clinical and Experimental Medicine, vol. 8, no. 4, pp. 6186-6192, 2015.

[26] M. Takahashi, Z. Chen, K. Watanabe et al., "Toll-like receptor 2 gene polymorphisms associated with aggressive periodontitis in Japanese," The Open Dentistry Journal, vol. 5, no. 1, pp. 190194, 2011.

[27] S. Schulz, L. Hierse, W. Altermann et al., “The del/del genotype of the nuclear factor-kappaB -94ATTG polymorphism and its relation to aggressive periodontitis," Journal of Periodontal Research, vol. 45, no. 3, pp. 396-403, 2010.

[28] G. Zhu, C. Li, Z. Cao, E. F. Corbet, and L. Jin, “Toll-like receptors 2 and 4 gene polymorphisms in a Chinese population with periodontitis," Quintessence International (Berlin, Germany: 1985), vol. 39, no. 3, pp. 217-226, 2008.

[29] Y. Hui, L. Mei, L. Ting, Z. Qing, and W. Z. Min, “The association between Toll-like receptor $2 / 4$ gene polymorphisms and the risk of chronic periodontitis," Chinese Journal of Geriatric Dentistry, vol. 9, no. 6, pp. 333-338, 2011.

[30] R. N. Jing, Z. G. Xun, and Z. Y. Pin, "Study on association between toll-like receptor 2,4 gene polymorphisms and periodontitis," J Clin Stomatol, vol. 22, no. 12, pp. 709-711, 2006.

[31] M. Folwaczny, J. Glas, H. P. Torok, O. Limbersky, and C. Folwaczny, "Toll-like receptor (TLR) 2 and 4 mutations in periodontal disease," Clinical and Experimental Immunology, vol. 135, no. 2, pp. 330-335, 2004. 
[32] R. M. Harbord, M. Egger, and J. A. C. Sterne, "A modified test for small-study effects in meta-analyses of controlled trials with binary endpoints," Statistics in Medicine, vol. 25, no. 20, pp. 3443-3457, 2006.

[33] M. Egger, G. D. Smith, M. Schneider, and C. Minder, "Bias in meta-analysis detected by a simple, graphical test," BMJ, vol. 315, no. 7109, pp. 629-634, 1997.

[34] M. X. Han, C. Ding, and H. M. Kyung, "Genetic polymorphisms in pattern recognition receptors and risk of periodontitis: evidence based on 12,793 subjects," Human Immunology, vol. 76, no. 7, pp. 496-504, 2015.

[35] G. G. Song, J. H. Kim, and Y. H. Lee, "Toll-like receptor (TLR) and matrix metalloproteinase (MMP) polymorphisms and periodontitis susceptibility: a meta-analysis," Molecular Biology Reports, vol. 40, no. 8, pp. 5129-5141, 2013.

[36] D. F. Lappin, S. Sherrabeh, and C. Erridge, "Stimulants of Tolllike receptors 2 and 4 are elevated in saliva of periodontitis patients compared with healthy subjects," Journal of Clinical Periodontology, vol. 38, no. 4, pp. 318-325, 2011.

[37] N. Buduneli, O. Ozcaka, and A. Nalbantsoy, "Salivary and plasma levels of Toll-like receptor 2 and Toll-like receptor 4 in chronic periodontitis," Journal of Periodontology, vol. 82, no. 6, pp. 878-884, 2011.

[38] A. S. Schaefer, G. M. Richter, M. Nothnagel et al., "A genomewide association study identifies GLT6D1 as a susceptibility locus for periodontitis," Human Molecular Genetics, vol. 19, no. 3, pp. 553-562, 2010.

[39] A. Ozturk and A. R. Vieira, "TLR4 as a risk factor for periodontal disease: a reappraisal," Journal of Clinical Periodontology, vol. 36, no. 4, pp. 279-286, 2009.

[40] S. H. Jin, X. Y. Guan, W. H. Liang, G. H. Bai, and J. G. Liu, "TLR4 polymorphism and periodontitis susceptibility: a meta-analysis," Medicine (Baltimore), vol. 95, no. 36, article e4845, 2016.

[41] P. Brennan, "Gene-environment interaction and aetiology of cancer: what does it mean and how can we measure it?," Carcinogenesis, vol. 23, no. 3, pp. 381-387, 2002. 